\title{
Veränderungen motorischer Funktionen bei psychiatrischen Erkrankungen
}

B ei Patienten mit psychiatrischen Erkrankungen findet sich von einzelnen neurologischen Ausfällen über Anomalien von Reflexen bis hin zu Auffälligkeiten komplexer Bewegungs- und Verhaltensstrukturen ein breites Spektrum veränderter motorischer Funktionen. Dieses Schwerpunktheft möchte einen Überblick über den neuesten Stand des Wissens in diesem Gebiet vermitteln. Besonders soll herausgearbeitet werden, welche Bedeutung den Veränderungen motorischer Funktionen heute für pathophysiologische Erklärungsmodelle, Diagnostik und Therapie bei psychiatrischen Erkrankungen zukommt.

Neuroleptika-induzierte, extrapyramidale Bewegungsstörungen stellen hinsichtlich ihrer Erklärungsmodelle oder der Relation zu Funktionseinschränkungen bei schizophrenen Patienten eine klinische Herausforderung dar. M. Dose stellt in seinem Beitrag den Bezug zu dem Einsatz neuentwickelter, ,atypischer“ Neuroleptika her. Das rechtzeitige Erkennen und Behandeln neuroleptisch bedingter, extrapyramidaler Nebenwirkungen ist für das Wohlbefinden, die Lebensqualität und Kooperationsbereitschaft der Patienten im Rahmen einer rezidivprophylaktischen Langzeitbehandlung weiterhin von großer Bedeutung.

Motorische Störungen bei schizophrenen Patienten können sich als Neuroleptika-induzierte Bewegungsstörungen, katatone Symptome und diskrete motorische Anomalien, sog. neurologische Soft Signs, manifestieren. In dem Beitrag von T. Jahn werden neue Befunde zu Prävalenz und Ursachen, neue, instrumentelle Analysemethoden und motorische Soft Signs als faszinierender Gegenstand der ätiologischen Grundlagenforschung beschrieben.

Störungen des Gangbildes sind ein häufiges Symptom psychiatrischer Erkrankungen. M. Perfahl und A. Putzhammer stellen in ihrem Beitrag verschiedene Untersuchungsmethoden zur Erfassung von Gangstörungen vor und gehen auf Studien zu Veränderungen des Gangbildes bei Patienten mit depressiven Störungen und die Parallelen zu Befunden bei Patienten mit Morbus Parkinson ein.

Mimischer Ausdruck spielt als motorische Funktion eine zentrale Rolle für Vermittlung und Erkennung von Emotionen. G. Juckel beschreibt in seinem Beitrag das Problem, ob und wie der emotionale Aspekt von dem rein motorischen Aspekt differenziert werden kann. Subklinische Auffälligkeiten bei depressiven Patienten sowohl unwillkürlicher als auch der willkürlicher Bewegungen haben Ähnlichkeiten mit Befunden bei Patienten mit Morbus Parkinson.

Störungen der Impulskontrolle, ein Merkmal vieler psychiatrischer Erkrankungen, sind durch Veränderungen biologischer, sozialer, kognitiver und motorischer
Dimensionen charakterisiert. In einem Beitrag aus Befunden der eigenen Arbeitsgruppe wird auf die zentrale Aufgabe des menschlichen Gehirns eingegangen, emotionale Reize in adäquates Verhalten umzusetzen. Untersuchungen an Patienten mit Borderline-Persönlichkeitsstörungen zeigen einen deutlichen Zusammenhang zwischen Impulsivität und Bewegungsprofilen, die auf Veränderungen zentralnervöser Bewegungsprogramme hindeuten, und bei Patienten mit Impulskontrollstörungen praktisch-klinische Bedeutung für forensische, diagnostische und therapeutische Fragestellungen haben.

Psychogene Bewegungsstörungen stellen in der Neurologie und Psychiatrie häufig eine Herausforderung für die behandelnden Ärzte dar. H. Ebel und C. Algermissen beschreiben in ihrem Beitrag die Bedeutung dieser Erkrankungen und gehen dann speziell auf verschiedene dissoziative Bewegungsstörungen ein, wobei die Wichtigkeit der interdisziplinären neurologischen und psychiatrischen diagnostischen Abklärung betont wird. Zur Therapie werden unter anderem kognitiv-behaviorale Techniken und Biofeedback eingesetzt.

Psychiatrische Erkrankungen manifestieren sich in Veränderungen des Erlebens und Verhaltens der betroffenen Patienten. Während das subjektive Erleben lediglich indirekt z.B. über verbale Äußerungen der Betroffenen zugänglich ist, lassen sich Änderungen des Verhaltens beobachten, erfassen, analysieren und quantifizieren. Die in diesem Rahmen auftretenden Veränderungen motorischer Funktionen sind externer Beobachtung und Messung zugänglich und spielen bei unterschiedlichen Krankheitsbildern eine Rolle für pathogenetische Modelle und in der klinischen Praxis für Diagnose und Therapie.

Besonderer Dank gilt den Mitautoren für ihr Engagement, mit ihren kompetenten Beiträge an der Entstehung dieses Heftes mitzuwirken.

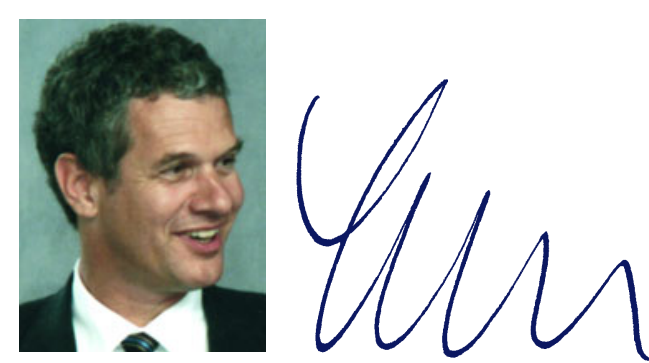

Priv.-Doz. Dr. med. Matthias R. Lemke, Bonn 